\title{
Accelerating parameter estimation of gravitational waves from black hole binaries with reduced order quadratures
}

\author{
Michael Pürrer*1,5, Rory Smith ${ }^{2}$, Scott Field $^{3}$, Priscilla Cañizares ${ }^{4}$, Vivien Raymond ${ }^{5}$, \\ Jonathan Gair ${ }^{4}$ and Mark Hannam ${ }^{1}$ \\ ${ }^{1}$ School of Physics and Astronomy, Cardiff University, Queens Building, CF24 3AA, UK \\ *E-mail: Michael.Puerrer@astro.cf.ac.uk \\ ${ }^{2}$ LIGO, California Institute of Technology, Pasadena, CA 91125, USA \\ ${ }^{3}$ Center for Radiophysics and Space Research, Cornell University, Ithaca, NY 14853, USA \\ ${ }^{4}$ Institute of Astronomy, Madingley Road, Cambridge, CB3 OHA, United Kingdom \\ 5 Max-Planck-Institut für Gravitationsphysik, Am Mühlenberg 1, 14476 Golm, Germany
}

\begin{abstract}
The inference of binary parameters from gravitational waves $(\mathrm{GW})$ is one of the key science goals of the collaboration operating the advanced ground-based LIGO-Virgo detector network. We employ reduced order quadratures (ROQs) to substantially reduce the size of large inner products arising in Bayesian parameter estimation (PE) and thus enable studies of the GWs emitted by coalescences of spinning stellar mass black hole binaries approaching the full design sensitivity of these detectors. We build the first ROQs that include the inspiral, merger and ringdown parts of the GWs for a single-spin precessing phenomenological waveform model (IMRPhenomP) and for an aligned-spin effective-one-body model (SEOBNRv2). The ROQs for SEOBNRv2 use a separate reduced order model (ROM) as a proxy. The ROQs we have constructed are suitable for any power spectrum density function of ground-based GW detector noise. We find speedups in the calculation of inner products and the likelihood function of up to several hundreds, reducing to days analyses which could otherwise take up to a year to complete.

Keywords: Gravitational waves; black hole binaries; reduced order modeling; reduced order quadratures; phenomenological; waveform model; effective-one-body.
\end{abstract}

\section{Introduction}

The ground-based advanced gravitational wave detectors advanced LIGO and advanced VIRGO will start taking data later this year. Coalescing black hole binaries are one of the most promising astrophysical sources. The emitted GW signal consists of a long chirp-like inspiral part where the amplitude and frequency both rise, the merger stage where the amplitude peaks, and the quasi-normal mode ringdown of the remnant Kerr black hole. To identify such highly structured signals the detector data is correlated via matched filtering against waveform model templates over the 15-dimensional and correlated parameter space which can be split into intrinsic (masses and spins of the binary) and extrinsic parameters which depend on the detector. Searches perform this filtering over a pre-built bank of templates and aim to identify loud events while parameter estimation (PE) looks to infer the physical parameters of candidate events with high accuracy.

$\mathrm{PE}$ requires up to several million evaluations of inner products between the data stream $d(t)$ and waveform templates $h(\vec{\lambda} ; t)$ as a function of physical parameters $\vec{\lambda}$ and time $t$. The data stream is the sum of a signal $s(t)$ and instrumental noise $n(t)$. 
Matched filtering is often performed in the Fourier domain, using the inner product

$$
\langle d \mid h(\vec{\lambda})\rangle=4 \operatorname{Re} \sum_{i=1}^{n} \frac{\tilde{d}^{*}\left(f_{i}\right) \tilde{h}\left(\vec{\lambda} ; f_{i}\right)}{S_{n}\left(f_{i}\right)} \Delta f_{i}
$$

where $S_{n}(f)$ is the power-spectral density of the detector noise, $\Delta f_{i}$ is the frequency spacing and $\tilde{h}(f)$ denotes the Fourier transform of $h(t)$. Even for a single matched filter, the computational cost of (a) generating a waveform template and (b) discretely evaluating a inner product can be very expensive.

Reduced order modeling $(\mathrm{ROM})^{1,2}$ has been shown to accelerate the evaluation of waveform models by factors of several thousand for effective-one-body waveforms ${ }^{3}$ and has been instrumental in making it possible to use these models for template bank generation and PE studies ${ }^{4}$. ROMs (or surrogate models) use reduced bases $(\mathrm{RB})$ built either with a greedy algorithm ${ }^{1}$ or the SVD and a set of sparse evaluation points ${ }^{2}$. The bases are built either for the complex waveform strain ${ }^{1}$ or for its amplitude and phases ${ }^{2}$. To construct a model an interpolant or fit needs to be constructed that allows one to obtain expansion coefficients for any desired physical parameter vector $\vec{\lambda}$. This is done either with the empirical interpolation method $^{1}$ that relies on the greedy $\mathrm{RB}$ or by interpolating projection coefficients of a grid of waveforms w.r.t. the RB, e.g. using tensor product splines ${ }^{2}$.

Reduced order quadratures (ROQ) ${ }^{6,7}$ accelerate the computation of inner products by replacing a standard Riemann sum discretization Eq. (1) with a quadrature rule that is tailored to the data stream. To build an ROQ a RB and empirical interpolant (EI) of the waveform strain is needed and, in practice, the evaluation of the strain must be a fast to compute expression. In particular, the ROQ can be used to build an accelerated likelihood function for Bayesian PE of GW signals ${ }^{8}$.

\section{Reduced Order Quadratures}

A RB $\left\{e_{i}\left(f_{j}\right)\right\}_{i=1}^{m}$ over a frequency grid $\mathcal{G}:=\left\{f_{j}\right\}_{j=0}^{n}$ for the Fourier domain waveform $\tilde{h}(\vec{\lambda} ; f)$, orthonormal w.r.t. to the discrete inner product $\langle\cdot \mid \cdot\rangle$ defined in Eq. (1), allows us to write a waveform $h$ as its projection $\mathcal{P} h$ onto the basis

$$
\tilde{h}\left(\vec{\lambda} ; f_{i}\right) \approx \mathcal{P} h:=\sum_{j=1}^{m}\left\langle h, e_{j}\right\rangle e_{j}\left(f_{i}\right) \quad i=0, \ldots, n .
$$

An efficient grid $\mathcal{G}$ can be chosen so that the spacing $\Delta f_{i}$ varies appropriately with the density of GW cycles as a function of frequency.

One can define a unique EI $\mathcal{I}[h]$ of a waveform $h$

$$
h\left(\vec{\lambda} ; f_{i}\right) \approx \mathcal{I}[h]\left(\vec{\lambda} ; f_{i}\right)=\sum_{j=1}^{m} c_{j}(\vec{\lambda}) e_{j}\left(f_{i}\right)=\sum_{j=1}^{m} B_{j}\left(f_{i}\right) \tilde{h}\left(\vec{\lambda} ; F_{j}\right) \quad i=0, \ldots, n,
$$

where $B_{j i} \equiv B_{j}\left(f_{i}\right)=\sum_{k=1}^{m} e_{k}\left(f_{i}\right) V_{k j}^{-1}, V_{i j}=e_{i}\left(F_{j}\right)$ and the empirical frequencies $F_{j} \subset \mathcal{G}$ are found from the solution of an $m \times m$ linear system $V_{i j} c_{j}(\vec{\lambda})=\tilde{h}\left(\vec{\lambda} ; F_{i}\right)$. 
The EI can be used to accelerate the computation of the inner product $\langle d \mid h(\vec{\lambda})\rangle$ defined in Eq. (1) between the data stream $d$ and a waveform template $h(\vec{\lambda})$ by replacing the template with its EI

$$
\langle d \mid h(\vec{\lambda})\rangle \approx\langle d \mid h(\vec{\lambda})\rangle_{\mathrm{ROQ}}=4 \operatorname{Re} \sum_{j=1}^{m} \sum_{i=1}^{n} \frac{\tilde{d}^{*}\left(f_{i}\right) B_{j}\left(f_{i}\right) \tilde{h}\left(\vec{\lambda} ; F_{j}\right)}{S_{n}\left(f_{i}\right)} \Delta f_{i} .
$$

We can precompute quadrature weights

$$
w_{j}=\sum_{i=1}^{n} \frac{\tilde{d}^{*}\left(f_{i}\right) B_{j}\left(f_{i}\right)}{S_{n}\left(f_{i}\right)} \Delta f_{i}
$$

for the given data stream $d$ and write the ROQ inner product as

$$
\langle d \mid h(\vec{\lambda})\rangle_{\mathrm{ROQ}}=4 \operatorname{Re} \sum_{j=1}^{m} w_{j} \tilde{h}\left(\vec{\lambda} ; F_{j}\right)
$$

Let $N$ be the size of the equally-spaced frequency grid $\mathcal{G}_{e}$ with the minimal spacing $\Delta f_{0}$ in $\mathcal{G}$. We find significant speedups $N / m$ for the evaluation of the inner product for low mass systems where $N \gg m$. We build RBs so that the EI error is at worst $\sim 10^{-6}$, well below calibration uncertainty of waveform models. In the presence of noise the error of interpolating the $\mathrm{RB}$ onto $\mathcal{G}_{e}$ also needs to be controlled.

\section{Status of ROQs for Inspiral-Merger-Ringdown Waveforms}

\subsection{ROQ for single-spin precessing phenomenological model}

The strain response $h(t)=F_{+} h_{+}(t)+F_{\times} h_{\times}(t)$ of a GW detector to an incoming signal depends linearly on the two polarizations $h_{+, \times}(t)$ of the signal, where $F_{+, \times}$specify the orientation of the detector to the binary. The strain enters the likelihood ${ }^{8}$, $\log \mathcal{L}(d \mid h(\vec{\lambda}))=\langle d \mid h\rangle-\frac{1}{2}[\langle h \mid h\rangle+\langle d \mid d\rangle]$, linearly in $\langle d \mid h(\vec{\lambda})\rangle$ and quadratically in $\langle h(\vec{\lambda}) \mid h(\vec{\lambda})\rangle$. Therefore, we build reduced bases for the individual polarizations and their four quadratic combinations.

We build ROQs for the "IMRPhenomP" 9 model that describes BH binary systems with single aligned and precessing spins. Model parameters include mass-ratio $q=m_{1} / m_{2}$, effective aligned spin $\chi_{\text {eff }}=\left(m_{1} \chi_{1}+m_{2} \chi_{2}\right) /\left(m_{1}+m_{2}\right)$, averaged spin in the orbital plane $\chi_{p}$, the angle $\theta_{\mathrm{JN}}$ between the total angular momentum $\vec{J}$ and the line of sight $\vec{N}$ of the observer to the binary, and an initial azimuthal phase $\alpha_{0}$.

We build a basis on the parameter space: $2 \leq M\left(M_{\odot}\right) \leq 20,1 \leq q \leq 8$, $-0.7 \leq \chi_{p} \leq 0.7,-0.7 \leq \chi_{\text {eff }} \leq 0.7,0 \leq \theta_{J} \leq 2 \pi, 0 \leq \alpha_{0} \leq 2 \pi$ and frequencies in the range $15 \leq f(\mathrm{~Hz}) \leq 4096$. The training set comprised about 20 million waveforms. The results for the bases are summarized in Table 1.

\subsection{ROQ for spin-aligned effective-one-body model}

We build ROQs for the spin-aligned effective-one-body model "SEOBNRv2" 3 by using a new ROM ${ }^{5}$ using extensions of the methods in Ref. 2. The ROQs were built 
Table 1. Reduced bases for IMRPhenomP for low frequency cutoffs $f_{\text {low }} \cdot m$ and $m^{\prime}$ are the dimension of the RBs for the polarizations and squared combinations, respectively.

\begin{tabular}{lllllll}
\hline$f_{\text {low }}$ & $f_{\text {high }}$ & $\Delta f_{0}$ & $m$ & $m^{\prime}$ & $N$ & Speedup $N /\left(m+m^{\prime}\right)$ \\
\hline $40 \mathrm{~Hz}$ & $4096 \mathrm{~Hz}$ & $1 / 64 \mathrm{~Hz}$ & 2770 & 1000 & $2.6 \times 10^{5}$ & 69 \\
$20 \mathrm{~Hz}$ & $4096 \mathrm{~Hz}$ & $1 / 256 \mathrm{~Hz}$ & 4593 & 2200 & $1.0 \times 10^{6}$ & 154 \\
$15 \mathrm{~Hz}$ & $4096 \mathrm{~Hz}$ & $1 / 512 \mathrm{~Hz}$ & 6247 & 3854 & $2.1 \times 10^{6}$ & 206 \\
\hline
\end{tabular}

on the 4-dimensional parameter space $\left\{M_{c}=M \eta^{3 / 5}, \eta=q /(1+q)^{2}, \chi_{1}, \chi_{2}\right\}$ where $M_{c} \in[1.2,17.4] M_{\odot}, \eta \in[0.05,0.25]$, and $\chi_{i} \in[-1,0.99]$. Guided by the spacing used in the ROM we generate a training set of about 3 million configurations on the boundary of the parameter space hypercube. To obtain a compact basis and reduce memory overheads for the basis construction we use unequally spaced frequency grids with the frequency spacing changing according to the density of cycles. In Table 2 we summarize results for the reduced bases for 10, 20, and $40 \mathrm{~Hz}$ starting frequency.

Table 2. Reduced bases for SEOBNRv2 for low frequency cutoffs $f_{\text {low }}$.

\begin{tabular}{lllllll}
\hline$f_{\text {low }}$ & $f_{\text {high }}$ & $\Delta f_{0}$ & $m$ & $n$ & $N$ & Speedup $N / m$ \\
\hline $40 \mathrm{~Hz}$ & $4096 \mathrm{~Hz}$ & $1 / 64 \mathrm{~Hz}$ & 1613 & 3705 & $2.6 \times 10^{5}$ & 161 \\
$20 \mathrm{~Hz}$ & $4096 \mathrm{~Hz}$ & $1 / 256 \mathrm{~Hz}$ & 3049 & 10320 & $1.0 \times 10^{6}$ & 342 \\
$10 \mathrm{~Hz}$ & $4096 \mathrm{~Hz}$ & $1 / 1024 \mathrm{~Hz}$ & 7421 & 31230 & $4.2 \times 10^{6}$ & 564 \\
\hline
\end{tabular}

\section{Conclusion}

We will present GW applications for the ROQs discussed in Sec. 3 in future work.

\section{Acknowledgments}

We thank P. Hopkins for computational support. Computational resources were provided by the ARCCA, Stampede, SuperMike2, and Zwicky clusters. MH was supported by STFC grant ST/H008438/1 and MP, MH by ST/I001085/1.

\section{References}

1. S. Field, et al. PRX 4, 031006, 2014.

2. M. Pürrer, CQG 19, 195010, 2014.

3. A. Taracchini et al., PRD 89, 061502, 2014.

4. J. Veitch, and M. Pürrer, and I. Mandel, PRL 115, 141101, 2015.

5. M. Pürrer, arXiv:1512.02248,

6. P. Canizares, et al. PRD 87, 124005, 2013.

7. P. Canizares, et al. PRL 114, 071104, 2015.

8. J. Veitch et al., PRD 91, 042003, 2015.

9. M. Hannam et al., PRL 113, 151101, 2014. 